\title{
How Women with Endometriosis Experience Health Care Encounters
}

\author{
Agneta Pettersson, PhD, ${ }^{1}$ and Carina M. Berterö, $\mathrm{PhD}^{2, *}$
}

\begin{abstract}
Objective: The aim of this meta-synthesis was to synthesize and interpret the available qualitative studies to increase our understanding and extend knowledge about how women with endometriosis experience health care encounters.

Methods: The literature review was carried out using CINAHL, Psychinfo, Academic Search Premier, PubMed, and Scopus, from 2000 to 2018, and was limited to articles in English. Articles were only included if they reported original relevant research on endometriosis and women experiences.

Results: The meta-synthesis was based on 14 relevant studies. They included 370 women with diagnosed endometriosis, 16-78 years of age. Three fusions were identified and interpreted in this meta-synthesis. The first was: Insufficiency knowledge, where the physicians could judge the symptoms to be normal menstruation without examining whether there were other underlying causes. The second fusion was Trivializing — just a women's issue, where the physicians thought that the symptoms were part of being a woman, and women's' discomfort was trivialized or completely disregarded. The third fusion was Competency promotes health, where the insufficiency of knowledge became a minor concern if women had a supportive relationship with their physician and the physician showed interest in their problems.

Conclusions: Women with endometriosis experience that they are treated with ignorance regarding endometriosis in nonspecialized care. They experience delays in both their diagnosis and treatment and feel that health care professionals do not take their problems seriously. In addition, it appears that increased expertise and improved attitudes among health care professionals could improve the life situation of women with endometriosis.
\end{abstract}

Keywords: endometriosis; health care encounter; hermeneutics; meta-synthesis; qualitative research; women's experiences

\section{Introduction}

Endometriosis is a chronic, inflammatory, and estrogen-dependent gynecological disease associated with pain and infertility that affects women of reproductive age. ${ }^{1}$ Endometriosis has been here throughout the ages and strikes women worldwide. ${ }^{2}$ It is estimated that 176 million women worldwide may be living with endometriosis. Approximately $10 \%$ of women of reproductive age are affected. ${ }^{3}$ Endometriosis occurs when endometrial cells, which normally line the uterus, grow outside the uterus. This tissue implants in, and forms lesions on, other organs, including the ovaries, bowel, bladder, and the Pouch of Douglas. 4

Gynecological symptoms of endometriosis include, dysmenorrhea, dyspareunia, chronic pelvic pain, bleeding, fatigue, and, in some cases, urological or gastrointestinal symptoms (such as dysuria, dyschezia). Compromised fertility or infertility are other symptoms associated with endometriosis. ${ }^{5,6}$

${ }^{1}$ Swedish Agency for Health Technology Assessment and Assessment of Social Services (SBU), Stockholm, Sweden.

${ }^{2}$ Division of Nursing Science and Reproductive Health, Department of Health, Medicine and Caring Sciences, Faculty of Medicine, Linköping University, Linköping, Sweden.

*Address correspondence to: Carina M. Berterö, PhD, Division of Nursing Science and Reproductive Health, Department of Health, Medicine and Caring Sciences, Linköping University, 58183 Linköping, Sweden, E-mail: carina.bertero@liu.se

(c) Agneta Pettersson and Carina M. Berterö, 2020; Published by Mary Ann Liebert, Inc. This Open Access article is distributed under the terms of the Creative Commons License (http://creativecommons.org/licenses/by/4.0), which permits unrestricted use, distribution, and reproduction in any medium, provided the original work is properly cited. 
Laparoscopy is the most common procedure used to diagnose endometriosis, ideally confirmed by histology. ${ }^{7,8}$ Delay in the diagnosis of endometriosis may have several causes such as false diagnosis and normalization of symptoms, ${ }^{9}$ but delay often occur because the gold standard for disease confirmation is using laparoscopy and histology. Such delays can adversely affect reproductive potential and functional outcomes. ${ }^{8}$ Endometriosis affects a woman's life considerably. A recent systematic review, ${ }^{10}$ found only 18 qualitative articles on endometriosis. This review showed that endometriosis affected all areas of a woman's life; sex life, social life, and work life, and medical experiences, symptom, and infertility are in some way intertwined and added distress to life. ${ }^{10}$ This review also pointed out that there were few studies on women's experiences of endometriosis-associated infertility and of the impact of reduced social participation on perceived support and emotional wellbeing. There are also few systematic reviews or meta-analysis that address psychological care for women with endometriosis. ${ }^{11,12}$ These reviews focus on treatment or interventions that may be promising in reducing endometriosis pain, anxiety, depression, stress, and fatigue. Another area where there is little, or no research is how women with endometriosis experience health care encounters. Such knowledge is important for providing high-quality care. Therefore, the objective of this meta-synthesis was to synthesize and interpret the available qualitative studies to increase our understanding and extend knowledge about how women with endometriosis experience health care encounters.

\section{Materials and Methods}

This article is an update of a qualitative meta-synthesis conducted by the Swedish Council for Health Technology Assessment as part of a report on the diagnosis and treatment of Endometriosis. ${ }^{13}$ This is a qualitative meta-synthesis, that is, an interpretive integration of qualitative findings that offers more than the sum of the individual data sets because it provides an innovative interpretation of the separate findings. ${ }^{14-16}$ The new findings and conclusions are derived from examining all the articles in a sample as a collective group, presenting interpretations that are representative since it is based on several articles. ${ }^{14}$ Qualitative metasynthesis allows for a broader approach to evidencebased research and practice by expanding how knowledge can be generated and used in the researched area. ${ }^{17,18}$ A meta-synthesis is the outcome of a metadata method. It is a distinct approach to new inquiry based on critical interpretation of existing qualitative studies. ${ }^{16}$ "It creates a mechanism by which the nature of interpretation is exposed and the meanings that extend well below those presented in the available body of knowledge can be generated. As such, it offers a critical, historical and theoretical analytic approach to making sense of qualitative knowledge."16, p2 The review/meta-synthesis was not registered in the international prospective register of systematic reviews (PROSPERO), since the data were already extracted, and the analysis was conducted.

\section{Sample}

Relevant qualitative research studies were retrieved on two occasions. A comprehensive search in the bibliographic databases CINAHL, Psych info, Academic Search Premier, PubMed, and Scopus was conducted on August 30, 2017. The articles had to meet the following inclusion criteria: (1) have a focus on women with endometriosis; (2) make explicit references to the use of qualitative research/studies or mixed methods, where the qualitative findings were reported separately; (3) have a focus on women's perspectives and experiences of living with endometriosis and how they experienced the encounters with health care; (4) published from 2000 to August 2017 and written in English. Exclusion criteria were quantitative research and literature reviews. An additional, selective, search in PubMed was conducted in December 2018, using the search terms endometriosis, women's experiences, and health care encounters. As shown in the flowchart in Figure 1, 24 studies were retrieved for consideration. A complementary search identical with 2018 was performed in December 2019. No more studies were retrieved.

\section{Meta-method}

The meta-method requires an analysis of the rigor and soundness of the research methods used in each of the studies reviewed to determine the appropriateness of the methods. ${ }^{15}$ The meta-method procedure had two steps. First, the studies were evaluated, with an emphasis on research design and data collection methods, to ensure that the article met the study's inclusion criteria. ${ }^{16}$ Of the 54 articles, 30 were excluded. One of these evaluated a training program and used a survey with open-ended questions. This article was retained for validation of our results. Second, the studies were appraised; incorporating the reading guide for both an individual appraisal of each possible study considered for inclusion, together with comparative appraisal 


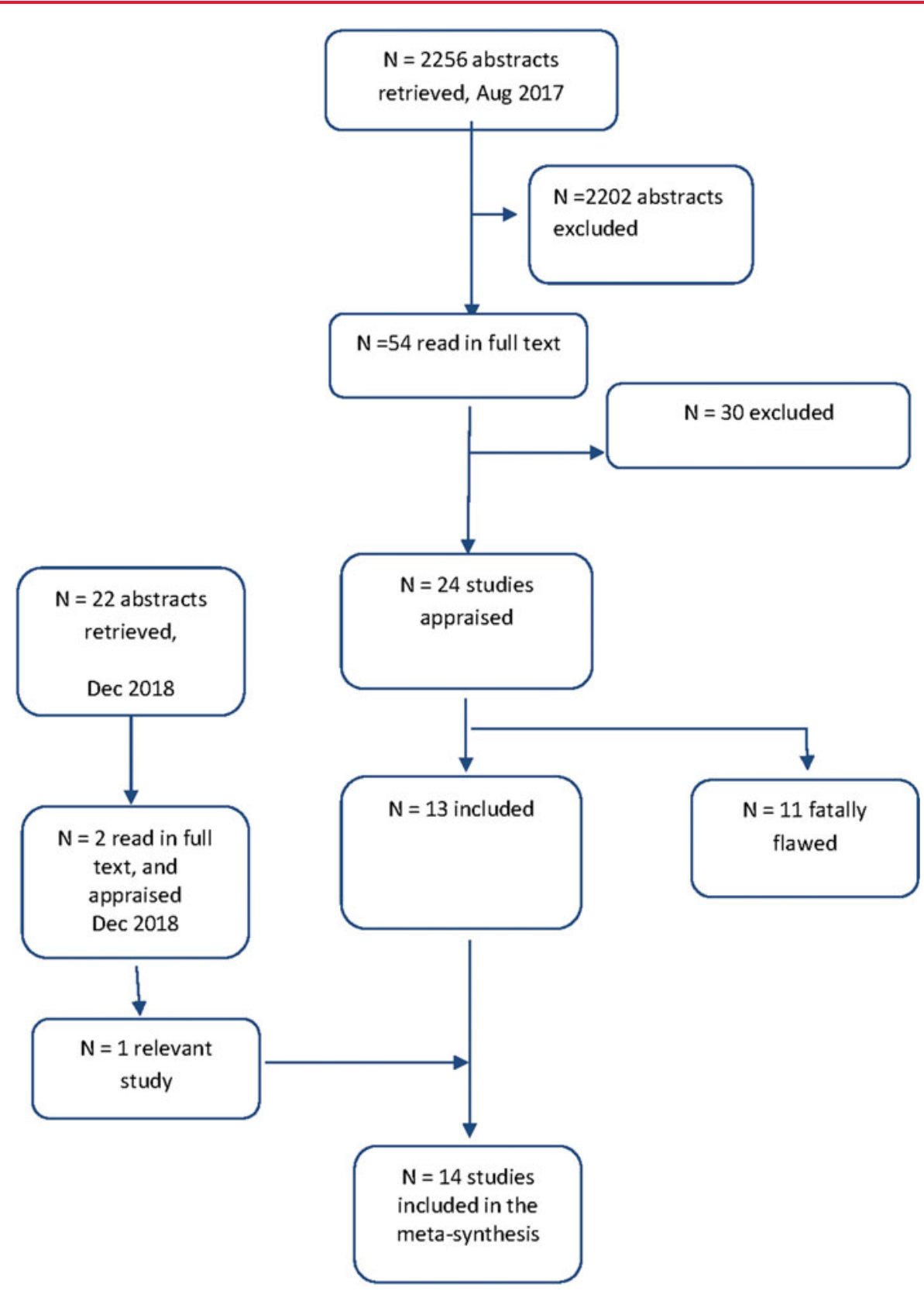

FIG. 1. Flowchart of the literature search process.

across studies. Key features of the studies were summarized, and a cross-study display was developed presenting the 14 articles selected for the study (Table 1). There is a large amount of data in each study, so the recommendation is that $10-12$ studies is an ideal number for a meta-synthesis. ${ }^{17}$ The studies represented the following disciplines: sociology, medicine, nursing, and physiotherapy (Table 1).
The size of the research sample reported in each article ranged from 9 to 74 , with a total sample size of 370 (mean sample size 26). The women in these studies were 16-78 years of age and represented different countries: Australia, ${ }^{18-21}$ Great Britain, ${ }^{22-25}$ Italy, ${ }^{26}$ New Zealand, ${ }^{27,28}$ South Africa, ${ }^{29}$ and Sweden. ${ }^{30,31}$ The data from the 370 women were based on individual interviews, in one case combined with focus group 


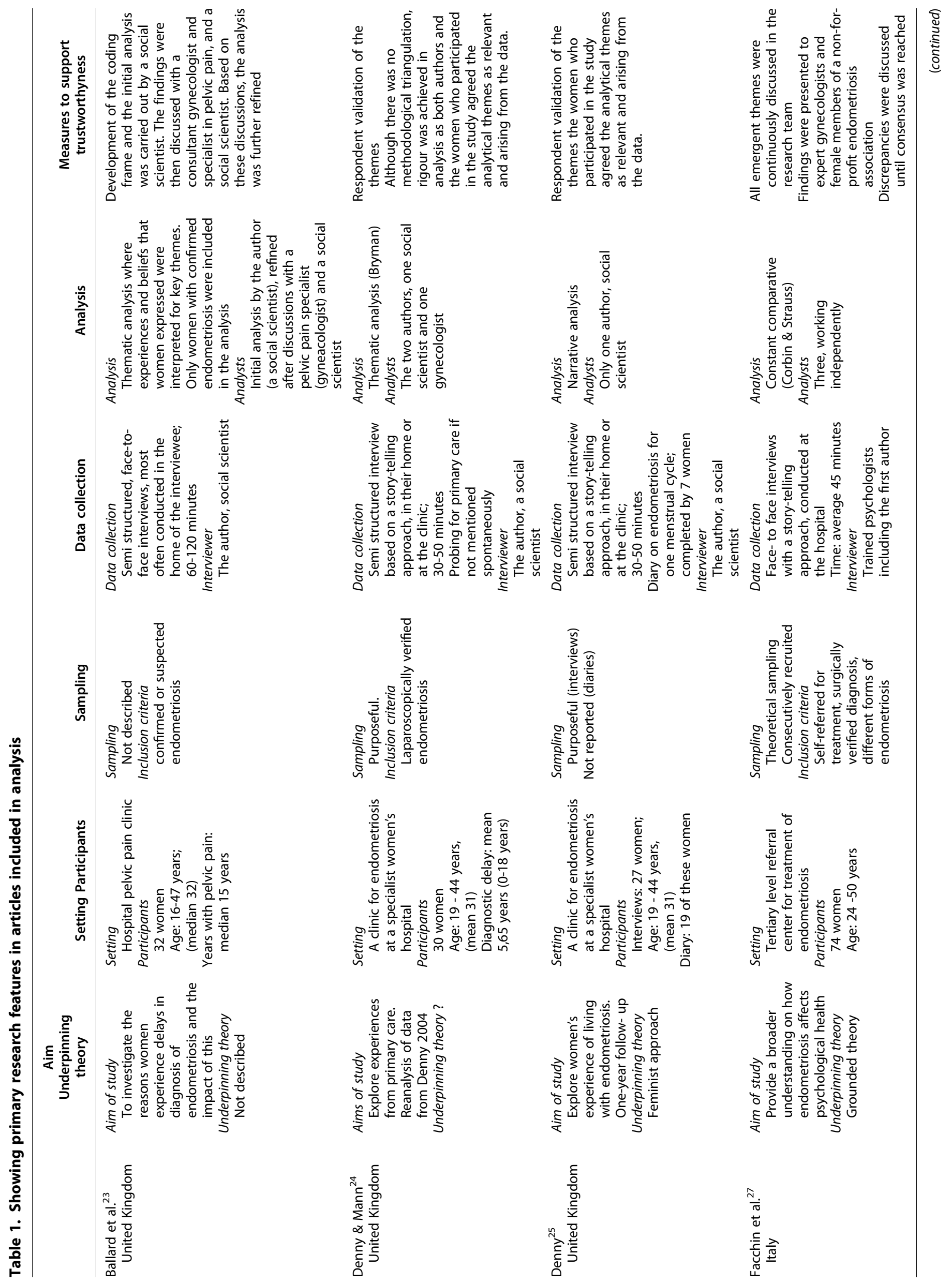




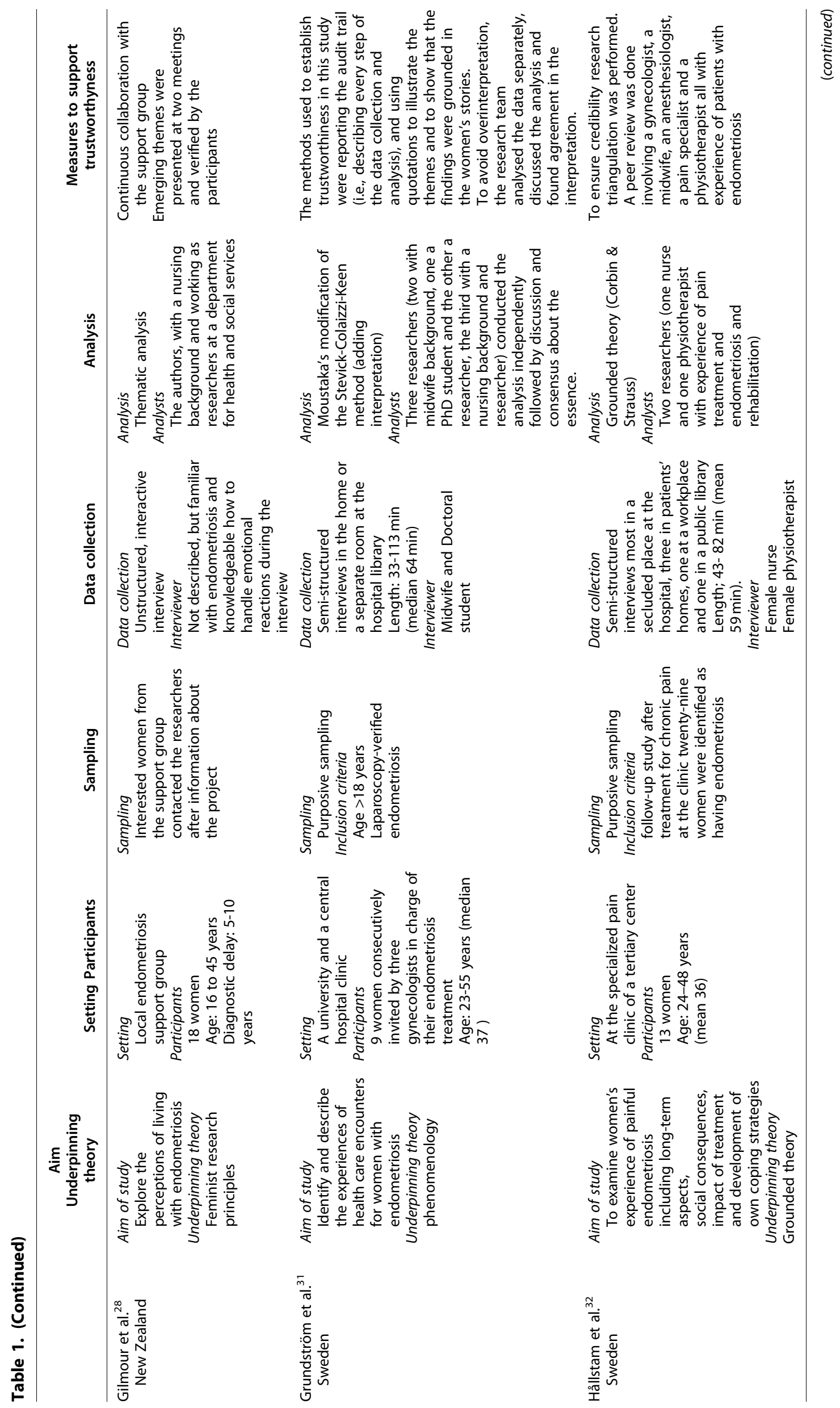




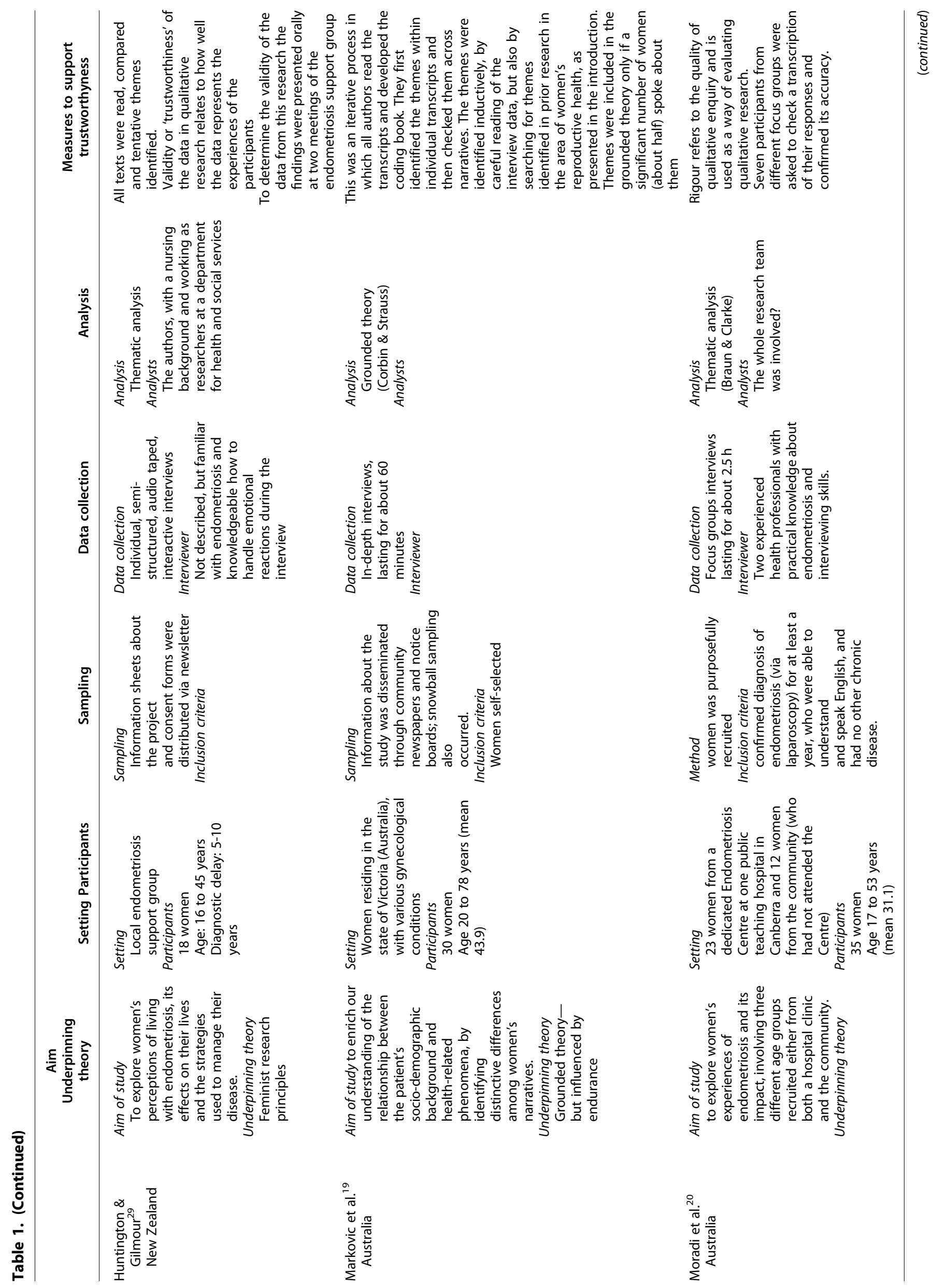




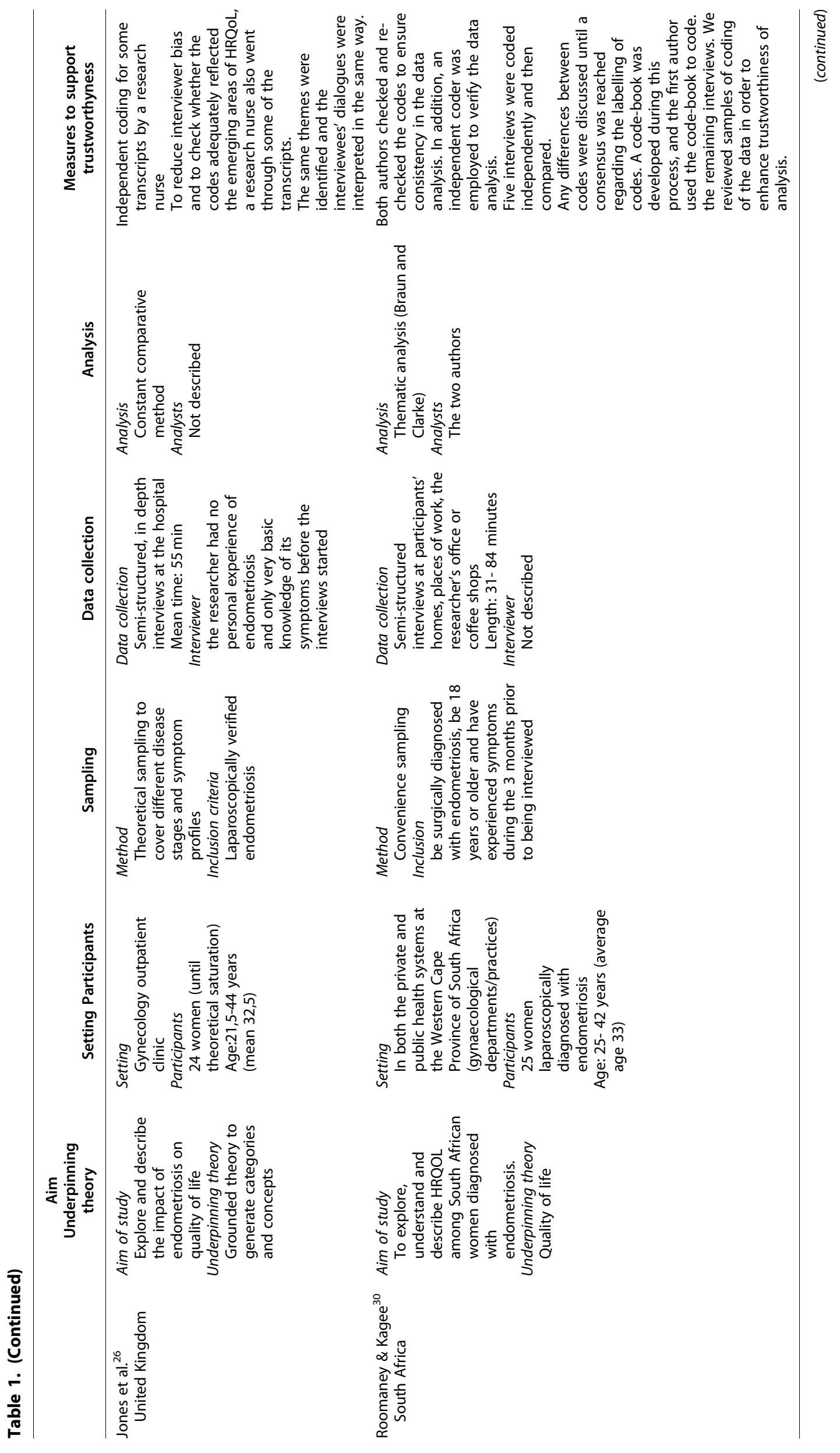




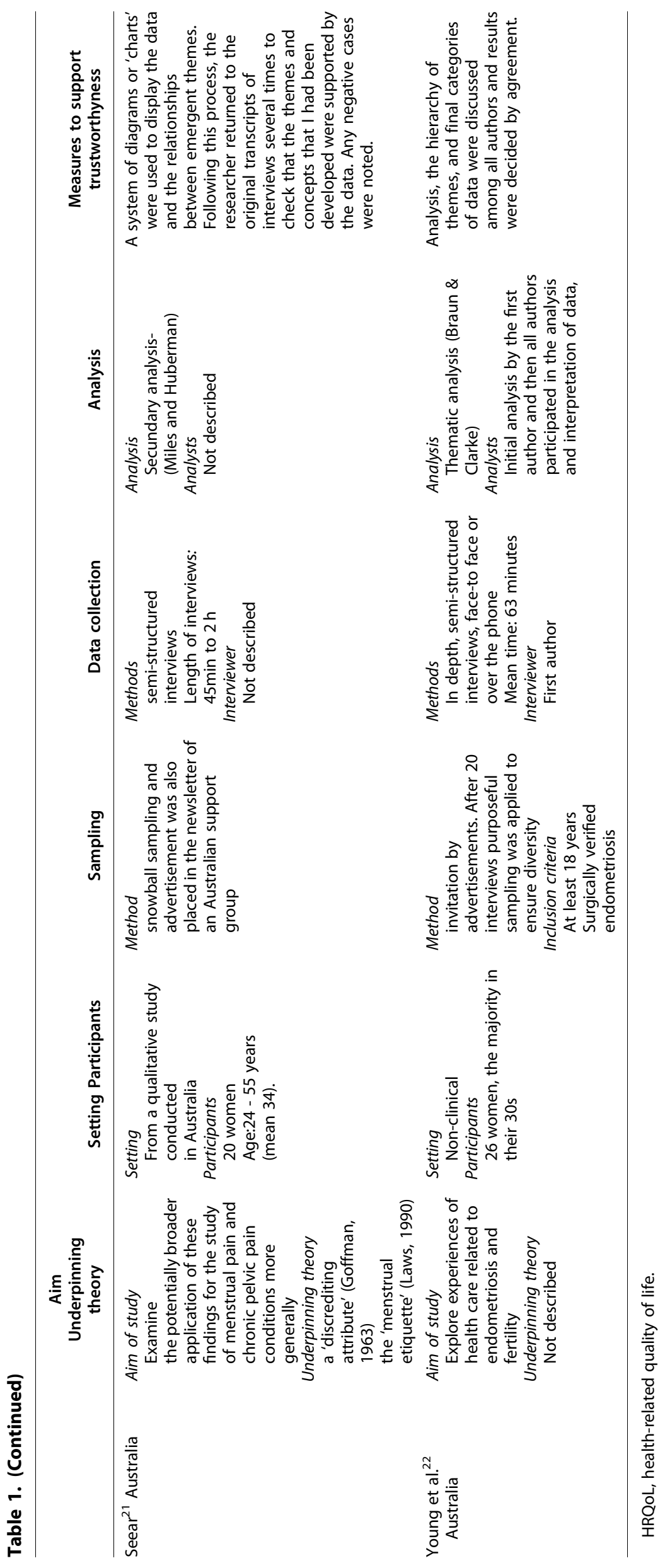


interviews, and one study was based on focus group interviews. All articles had been published from 2004 to 2018. In the second step, the appraisal process contributed to an understanding of how the methodology used in the original research had been applied to study a phenomenon and how that methodology had shaped the researcher's knowledge. The research designs of the primary research articles were compared and contrasted to identify the underlying assumptions of research methodologies, as well as the findings reported. ${ }^{13.15}$ The authors of the articles meeting the criteria for inclusion in this study described the methods used as qualitative, that is, the studies used phenomenology, narrativism, thematic analysis, grounded theory, and constant comparative analysis. Some researchers used thematic analysis and narrativism for secondary analysis. Analysis revealed that there was a variation in the rigor with which the tenets of the identified method were applied, as well as a range in the quality and quantity of direct quotes available to the analysts of this meta-synthesis.

\section{Preparing the findings for meta-synthesis}

The quality of the way in which findings were presented in the articles varied; most articles presented raw data as thematic surveys and/or direct quotations from participant interviews. The metadata analysis used the technique of hermeneutic appraisal ${ }^{33}$ to extract statements from the articles' findings to evaluate the horizon of the text. The analysts then interpreted these statements within the context of a guiding question: Is this about being perceived or about the health care encounter. Working inductively from these interpretations, the researchers were able to identify possible fusions. ${ }^{15,16}$

\section{Results}

The meta-synthesis resulted in three fusions: Insufficient knowledge, Trivializing-just a women's issue, and Competence promotes health.

\section{Insufficient knowledge}

The women often stated that they had repeated problems that the cause of the symptoms seemed to be unknown and they had not been diagnosed. The women felt that the physicians lacked knowledge of the disease, which led to the diagnosis being delayed and that they did not receive adequate treatment. The insufficient knowledge could be demonstrated in several ways. The physicians could judge the symptoms as normal menstruation without examining whether there were other underlying causes. The physicians were annoyed when the women pointed out that their symptoms were similar to those of other women with diagnosed endometriosis. In other cases, incorrect diagnoses were made. The handling of the women's case, both before and after diagnosis, was often based on medical myths. ${ }^{19-32}$ In this fusion of insufficient knowledge, three themes were identified: normalization of pain as menstrual pain, incorrect diagnosis, and treatment was decided based on medical myths.

The women felt that the physicians dismissed their pain as a menstrual pain and as such something that was normal and that was part of being a woman, something all women live with. The women might receive the comment that they were unlucky that they belonged to the group of women who have severe menstrual pain. It also happened that the physicians believed that the women did not know what pain was (not experienced real pain) and therefore did not tolerate ordinary menstrual pain, even though the women had problems going to the toilet, having intercourse, etc. ${ }^{19,20,23-25,27,29,31,32}$

The normalization meant that physicians usually did not search for some other underlying cause of the pain. Instead, they often focused on the relief of symptoms. Sometimes lifestyle changes were recommended for example, physical exercise, and the physicians prescribed contraceptive pills to reduce bleeding and pain, even to very young teenage girls. Sometimes the physicians prescribed pain killers and sometimes the women themselves had to suggest the type of pain relief that suited them. Some women refused to take birth control pills because they wanted to know what caused their pain. By extension, the normalization led to the diagnosis being delayed. ${ }^{18,20,22-26,28,30,31}$

If the women's problem remained after treatment with painkillers and contraceptive pills, the physicians looked for simple causes. The physicians, however, were not inclined to accept that women's symptoms could be caused by any gynecological disorder or illness. Instead, the physicians gave other, incorrect diagnoses. The pain and the problem were located in the abdomen. Women who reported that they had simultaneous problems with the abdomen or intestines could receive diagnoses such as Irritable Bowel Syndrome, appendicitis, or muscle stretching. The problems could also be seen as mental. Some physicians thought the problem was in the head and not in the body. The women were diagnosed with depression or the physician blamed the symptoms on possible early abuse of 
alcohol or other drugs, on infection, or on previous miscarriage. Young women, students, were treated as being persons who did not take care of themselves. ${ }^{19,23,26,29-32}$

The women were referred to specialists such as psychiatrists or gastroenterologists before finally getting to meet a gynecologist. Some physicians put faith in socalled medical myths, which women knew were wrong, for example, that young women or teenage girls were too young to have endometriosis. Other myths concerned treatment when the women had been diagnosed. The women could receive the recommendation to remove the uterus, even though they were only in their twenties. Another myth that the physicians referred to was that the women's pains would be cured if they gave birth to children. Some arguments were that the hormones would restore the balance in the body or that the endometriosis would shrink and would not grow further. ${ }^{20,22,24,26,27,29,31,32}$

The recommendation for childbirth could be perceived as particularly offensive. Some women recalled that doctors recommended pregnancy as a treatment, even if they were infertile. Some other women were in such a situation that it was not suitable to have children at that time.

\section{Trivializing-just a women's issue}

The women in these studies felt that no one took their problems seriously. Their discomfort was trivialized or completely disregarded. The physicians meant/felt that the symptoms were part of being a woman. The women's problems did not generate much interest and did not lead to continued investigation. The attitude was to take some painkillers and live as usual.

The approach of the physicians was described as offensive and sometimes blameful. There was little understanding of the fact that the problems could affect women's social life and quality of life..$^{21,23-27,29,31}$

In this fusion of incorrect diagnoses three themes were identified: Not being taken seriously, no interest in women's problems, and insensitivity to women's situation.

The women felt that their symptoms were more difficult than painful menstruation and referred to their menstrual history and compared themselves with family and friends. The most common cause of dissatisfaction or anger by women was the feeling that physicians did not take their symptoms seriously. As their problems were not taken seriously, the physicians did not refer them for further investigation and treatment. The women felt denied since the physicians' attitude was that women exaggerated or imagined their symptoms, like having a form of "fantasy pain," or had low pain thresholds. The women were requested to learn to handle their pain. Other women felt that the physicians wanted the women to feel that they had failed morally because they could not cope with their pains like other women. There was always a struggle to be taken seriously. ${ }^{24-27,31,32}$

The women who, after years of rejection and normalization of the pain, were referred for examination and received a diagnosis felt that they had finally received "evidence" that they had a disease and thus were able to discuss treatment options. The diagnosis was a relief. It was a confirmation that they had a disease and it was not just something in their head. However, even when the disease was verified with, for example, image evidence, physicians could question whether the pains really were proportional to the size of the lesions. ${ }^{23-27,31,32}$

The women felt that their symptoms were not very interesting or exciting for the physicians, or as it was expressed in a study-"endometriosis is not a sexy disease." They felt invisible when the physicians were not interested in understanding them and their pain problems. Others felt that they were wasting their physicians' "time" because the physician believed that the trouble "was in the head". The physicians showed lack of interest and distance when the women talked about their problems. They sighed, drummed their fingers on the table, avoided eye contact, and responded monotonously or with specialist terms. When simple explanations or jargon did not work, the physicians switched to normalizing or trivializing the problems.

The most difficult thing was when the physician moved the burden over to the women by asking how they wanted to be helped. ${ }^{23-25,27,29-32}$

There were also physicians who in a more brutal way showed their lack of interest. The physicians could argue that there was no diagnosis called menstrual pain, and that it was only stupid women who expressed themselves like this. ${ }^{24-26,28,31,32}$

The physicians could show insensitivity to the women's situation, which could be experienced as verbally, psychologically, and physical violations. For example, the physician could convey the information about the woman's endometriosis and infertility in an insensitive manner. It could be positive to get all this important information about endometriosis, but if the physician in the same breath mentioned infertility, it became too heavy. When the women raised the issue of children, 
they were abruptly informed that they would probably need to adopt. The physicians had not endeavored to find out about the women's life situation. There were women who had always longed to create a family but who were given the message about their infertility in an insensitive way, and this affected them for a long time to come. Some women even considered suicide because they had always wanted to have a family, and this dream had been totally crushed. ${ }^{2-25,30-32}$

The women described that they were in a difficult and vulnerable situation, filled with anxiety and frustration, which was mentally devastating. It was then difficult to be called stupid, crazy, and mentally ill. $^{22,24,25,27,28,31,32}$

The women also felt physically vulnerable. Painful gynecological examinations could feel like abuse even though they were routine. The women did not want to undergo such examinations, but they had to.

\section{Competence promotes health}

The third fusion describes positive experiences of meetings with physicians, and four studies contributed. ${ }^{22,23,27,31,32}$ Although the women mostly experienced ignorance and lack of interest in primary care, there were physicians who were interested in their problems. The insufficiency of knowledge became a minor concern if the women had a supportive relationship with their physician. Such physicians could help by listening and not delaying the referral to a specialist. One woman also said that the physician was not aware of endometriosis during the first visit but had done his homework before the next meeting.

Transferring from general to specialized care could offer a positive change. The women were reassured by knowing that they would be treated by physicians with special skills and competency. After several years of suffering they received a diagnosis and an explanation of why they had these problems. They acknowledged that the gynecologists showed that they cared, actively listened, and took them seriously. Such dedicated gynecologists took the time to explain and provide relaxation tips that could make the examination procedure easier. Women with fertility problems reported that their disease burden was alleviated by the physician communicating well about fertility. It also meant a lot that the gynecologist shifted focus from only the disease to how it affected the woman's life and situation. This strengthened and promoted women's health, helping them to continue their lives.
Validation of the findings using a survey study The analysts used a large qualitative survey study, including open questions to validate or "member check" the findings and interpretations from the metadata analysis/synthesis. ${ }^{34}$ This survey study included 135 women with endometriosis who evaluated a training program about social support, treatment, as well as professional and health care system performance. Because our study is a meta-analysis/synthesis of text, we did not have the opportunity to "member check" the findings with participants of the study. Since this survey study was performed with other participants and in another country (Germany), ${ }^{35}$ the findings from this survey study can be used for validation and triangulation of this meta-synthesis. The findings of this survey study pointed out some important factors.

Knowledge and competence were found to be among the most important factors when making the women with endometriosis feel confident and cared for. The second factor of importance was that the physicians/gynecologist believed in the women's descriptions about the symptoms and problems, that is, the women were taken seriously. The care encounters should be imbued with understanding and empathy. The third factor that was experienced as supportive was open and clear communication, based on interest and sensitivity. Obstacles and hindrances for women with endometriosis concerning managing their situation were ignorance, insufficient empathy, and nonsensitivity. These findings are in great agreement with those in the meta-synthesis, which strengthens its trustworthiness.

\section{Discussion}

In this meta-synthesis, data from 14 research studies on women with endometriosis were reinterpreted to gain a deeper understanding of how they were perceived or about the health care encounter. We used the women's quotations presented as raw data and the text in the findings to draw an interpretation and gain an understanding of the phenomenon under study. It is interesting to note that even though the studies are from 2004 until 2018, the experiences of the women seem to remain. There are still the same issues and approaches perceived, but medical progresses are made.

The credibility (validity) of the data interpretations in this study is supported because they are the result of a systematic approach ${ }^{13}$ and can always be arbitrated 
for or against a variety of other interpretations. The data analyzed and interpreted in this study comprised primary research conducted on women from different cultures and contexts, but all having an endometriosis diagnosis. There are several other reviews that have synthesized qualitative research on how endometriosis affects women's lives and how they handle the problems. ${ }^{10,36-39}$ One of them focused on experiences of care and patient centering, ${ }^{37}$ whereas the others had a broader perspective. However, the selection of studies differs in part between our meta-synthesis and the other overviews. In addition, new studies have been added that are only included in our overview. The article by Dancet et al. ${ }^{38}$ also combined quantitative and qualitative articles, but in our meta-synthesis we only used qualitative articles. The fusions identified in our overview have not been highlighted as much in the other overviews. Some of our findings regarding experiences, how being approached from health care, are unique to women with endometriosis.

Insufficiency knowledge and its underlying themes could be found in the overview. ${ }^{10}$ This overview, however, emphasized mainly that the women sought knowledge and that the doctors compensated for their lack of knowledge by being more responsive. Insufficient knowledge was made visible in incorrect diagnoses and delayed diagnoses in three of the reviews, ${ }^{9,36,37}$ whereas the fourth found that it was about the lack of information.

The findings in this meta-synthesis may seem discouraging; the treatment of the women revealed perceived with ignorance about endometriosis and they experienced that their problems were trivialized; it was just a "women's issue." This fusion was a strong theme based on the women's statements in the various studies that we included. In the overview articles, this "woman issue" was described as normalization of the problems and damaged wellbeing ${ }^{10,38}$ and also a cause of delayed diagnostics due to normalization and trivialization of women's problems. ${ }^{37}$ Dancet et al. ${ }^{38}$ mentions what lies in the subthemes of this fusion, namely to respect women's problems and to let the women be trusted. Others seem to be shared with persons with other types of chronic pain, mainly musculoskeletal and where most studies capture experiences from primary care. One common experience is the sense of not being believed since the pain is not visible or measurable was, for example, captured in qualitative evidence syntheses about chronic pain conditions ${ }^{40}$ and focusing on pelvic pain. ${ }^{41}$ The hard work to get a medical diagnosis was also central. Focusing on these issues there are some suggestions for developing endometriosis and pain centers, by using patient navigators, and working multidisciplinary. ${ }^{42}$

Our third fusion that competence promotes health, which is included in this theme, is the only fusion that brings forth some positive aspects acknowledged in the analyzed studies. That competency promotes health is often mentioned in one or two sentences here and there in the overviews. ${ }^{10,36}$ The fact that our three fusions are not clearly found in the overview articles that we compare in this study may be because these (three out of four) are merely descriptive and reflect what we already know. The fourth review article used "interpretation," but on a combined material; quantitative and qualitative articles. This causes a methodological difficulty to extract material narratively/interpretatively from quantitative results.

We performed a meta-synthesis to, through interpretation, lift research results/findings to the next level and not only repeat what we already knew with some certainty. ${ }^{14}$ The meta-synthesis used in this study was grounded in hermeneutic theories. ${ }^{16,34}$ In this way, we clarified the women's experience of the encounter in endometrial care. We have interpreted and synthesized fusions and therefore it seemed relevant to discuss the results against other reviews-bringing forth synthesized data. Trying to broaden the one perspective view.

When we validated our fusions with the survey study, ${ }^{35}$ there was a finding showing that there are possibilities to make a positive difference regarding these women, by showing empathy and adopting a professional and competent approach. In a quite recently published article ${ }^{43}$ it was shown that medical education needs to equip physicians with the skills to acknowledge and incorporate women's knowledge of their bodies within the medical encounters. It was also highlighted that the women should be acknowledged and treated as partners in their health.

\section{Conclusion}

The present meta-synthesis demonstrates that the "journey of endometriosis care" takes its starting point in primary care and there is a struggle to convince health care professionals that the symptoms are not ordinary menstrual pain and bleeding. There was also a struggle in that discomfort was trivialized and seen as women's issue. Transferring from general to specialized care could offer positive changes. The women were reassured 
by knowing that they would be treated by physicians with special skills and competency.

\section{Clinical Implication}

Living with endometriosis and how one is perceived in the health care encounters have a great impact on these women's lives. The results of the present study highlight the importance of providing support for women who have endometriosis, so they are able to manage their everyday lives. Health care professionals need to be aware of endometriosis as a disease and be more sensitive for individual pain pattern among women, which could facilitate manageability. ESHRE $^{44}$ presents clinical practice guidelines regarding medical progression, but health care professionals need to make their own clinical decisions based on their competency, considering the circumstances, and collaborate with the women.

\section{Author Disclosure Statement}

No competing financial interests exist.

\section{Funding Information}

No funding was received for this study.

\section{References}

1. Johnson NP, Hummelshoj L, Adamson GD, et al. World Endometriosis Society consensus on the classification of endometriosis. Hum Reprod 2017;32:15-24.

2. Benagiano G, Brosens I. The history of endometriosis: Identifying the disease. Hum Reprod 1991;6:963-968.

3. Endometriosis World. World Statistics of Endometriosis, 2017. Available at: https://endometriosisworld.weebly.com/world-statistics.html Accessed June 25, 2020.

4. Burney RO, Giudice LC. Pathogenesis and pathophysiology of endometriosis. Fertil Steril 2012;98:511-519.

5. Vercellini P, Fedele L, Aimi G, et al. Association between endometriosis stage, lesion type, patient characteristics and severity of pelvic pain symptoms: A multivariate analysis of over 1000 patients. Hum Reprod 2007;22266-22271.

6. Mao AJ, Anastasi JK. Diagnosis and management of endometriosis: The role of the advanced practice nurse in primary care. J Am Acad Nurse Pract 2010;22:109-116.

7. Wykes CB, Clark TJ, Khan KS. Accuracy of laparoscopy in the diagnosis of endometriosis: A systematic quantitative review. BJOG 2004;111:12041212.

8. Albee RB, Jr, Sinervo K, Fisher DT. Laparoscopic excision of lesions suggestive of endometriosis or otherwise atypical in appearance: Relationship between visual findings and final histologic diagnosis. J Minim Invasive Gynecol 2008;15:32-37.

9. Hudelist G, Fritzer N, Thomas A, et al. Diagnostic delay for endometriosisd Germany: Causes and possible consequences. Hum Reprod 2012;27: 3412-3416.

10. Young K, Fisher J, Kirkman M. Women's experiences of endometriosis: A systematic review and synthesis of qualitative research. BMJ Sexual Rep Health 2015;41:225-234.

11. Gao J, Liu HQ, Wang Y, Shang YL, Hu F. Effects of psychological care in patients with endometriosis: A systematic review protocol. Medicine 98. Medicine (Baltimore) 2019;98:e14772.
12. Evans S, Fernandez S, Olive L, Payne LA, Mikocka-Walus A. Psychological and mind-body interventions for endometriosis: A systematic review. J Psychosomat Res 2019;124:109756.

13. SBU. Endometrios; Diagnostik, behandling och bemötande (Endometriosis, diagnosis, treatment and being perceived). Stockholm: Swedish Agency for Health Technology Assessment and Assessment of Social Services, 2018.

14. Sandelowski $M$, Barroso J. Creating metasummaries of qualitative findings. Nurs Res 2003;52:226-233.

15. Sandelowski M, Barroso J. Toward a metasynthesis of qualitative findings on motherhood in HIV-positive women. Res Nurs Health 2003;26:153170.

16. Paterson ML, Thorne SE, Canam C, Jillings $C$. Meta-study of qualitative health research. Practical guide to meta-analysis and meta-synthesis. London: Sage Publications Ltd., 2001.

17. Kent B, Fineout-Overhold E. Using meta-synthesis to facilitate evidencebased practice. Worldviews Evid-Based Nurs 2008;5:160-162.

18. Bondas T, Hall EO. Challenges in approaching metasynthesis research. Qual Health Res 2007;17:113-121.

19. Markovic M, Manderson L, Warren N. Endurance and contest: Women's narratives of endometriosis. Health: An Interdisciplinary. J Soc Study Health Illness Med 2008;12:349-367.

20. Moradi M, Parker M, Sneddon A, Lopez V, Ellwood D. Impact of endometriosis on women's 'lives; a qualitative study. BMC Womens Health 2014;14:123-134.

21. Seear K. The etiquette of endometriosis: Stigmatization, menstrual concealment, and the diagnostic delay. Soc Sci Med 2009;69:12201227.

22. Young K, Fisher J, Kirkman M. Endometriosis and fertility: Women's accounts of healthcare. Hum Reprod 2016;31:554-562.

23. Ballard K, Lowton K, Wright J. What's the delay? A qualitative study of women's experiences of reaching a diagnosis of endometriosis. Fertil Steril 2006;86:1296-1301.

24. Denny E, Mann $\mathrm{CH}$. Endometriosis and primary care consultation. Eur J Obstet Gynecol Reprod Biol 2008;139:111-115.

25. Denny E. I never know from one day to another how I will feel: Pain and uncertainty in women with endometriosis. Qual Health Res 2009;19:985995.

26. Jones $G$, Jenkinson C, Kennedy S. The impact of endometriosis upon quality of life: A qualitative analysis. J Psychosom Obstet Gynecol 2004;25: 123-133.

27. Facchin F, Saita E, Barbara G, Dridi D, Vercellini P. "Free butterflies will come out of these deep wounds": A grounded theory of how endometriosis affects women's psychological health. J Health Psych 2018;23:538 549.

28. Gilmour JA, Huntington A, Wilson HV. The impact of endometriosis on work and social participation. Int J Nurs Pract 2008;14:443-448.

29. Huntington A, Gilmour JA. A life shaped by pain: Women and endometriosis. J Clin Nurs 2005;14:1124-1132.

30. Roomaney R, Kagee A. Salient aspects of quality of life among women diagnosed with endometriosis: A qualitative study. J Health Psychol 2018; 23:905-991.

31. Grundström H, Alehagen S, Kjølhede P, Berterö C. The double-edged experience of healthcare encounters among women with endometriosis: A qualitative study. J Clin Nurs 2018;27:205-211.

32. Hållstam A, Stålnacke BM, Svensén C, Löfgren M. Living with painful endometriosis-A struggle for coherence. A qualitative study. Sex Reprod Healthcare 2018;17:97-102.

33. Gadamer HG. Truth and method. London: Sheds and Wards, 1975/2001.

34. Risser J. Reading the text. In: Silverman JH, ed. Gadamer and hermeneutics. London: Routledge, 1991:93Y105.

35. Kundu S, Wildgrube J, Schippert C, Hillemanns P, Brandes I. Supporting and Inhibiting factors when coping with endometriosis from the patients' perspective. Geburtsh Frauenheilk 2015;75:462-469.

36. Culley L, Law C, Hudson N, et al. The social and psychological impact of endometriosis on women's lives: A critical narrative review. Hum Reprod Update 2013;19:625-639.

37. Kaatz J, Solari-Twadell PA, Cameron J, Schultz R. Coping with endometriosis. J Obstet Gynecol Neonatal Nurs 2010;39:220-225; quiz 225-226.

38. Dancet EA, Apers S, Kremer JA, Nelen WL, Sermeus W, D' Hooghe TM. The patient-centeredness of endometriosis care and targets for 
improvement: A systematic review. Gynecol Obstet Invest 2014;78: 69-80.

39. Erwin EJ, Brotherson MJ, Summers JA. Understanding qualitative metasynthesis. Issues and opportunities in early childhood intervention research. J Early Interv 2011;33:186-200.

40. Crowe M, Whitehead L, Seaton $P$, et al. Qualitative meta-synthesis: The experience of chronic pain across conditions. J Adv Nurs, 2017;73:10041016.

41. Toye F, Seers K, Hannink E, Barker K. A mega-ethnography of eleven qualitative evidence syntheses exploring the experience of living with chronic non-malignant pain. BMC Med Res Methodol 2017;17:116.

42. Opoku-Anane J, Orlando MS, Lager J, et al. The development of a comprehensive multidisciplinary endometriosis and chronic pelvic pain center. J Endometr Pelvic Pain Disord 2020;12:3-9.

43. Young K, Fisher J, Kirkman M. Partners instead of patients: Women negotiating power and knowledge within medical encounters for endometriosis. Fem Psychol 2020;30: 22-41.
44. Dunselman GA, Vermeulen N, Becker C, et al. ESHRE guidline: Management of women with endometriosis. Hum Reprod 2014;29: 400-412.

Cite this article as: Pettersson A, Berterö CM (2020) How women with endometriosis experience health care encounters, Women's Health Report 1:1, 529-542, DOI: 10.1089/whr.2020.0099.

\section{Abbreviation Used}

$\mathrm{HRQoL}=$ health-related quality of life
Publish in Women's Health Reports

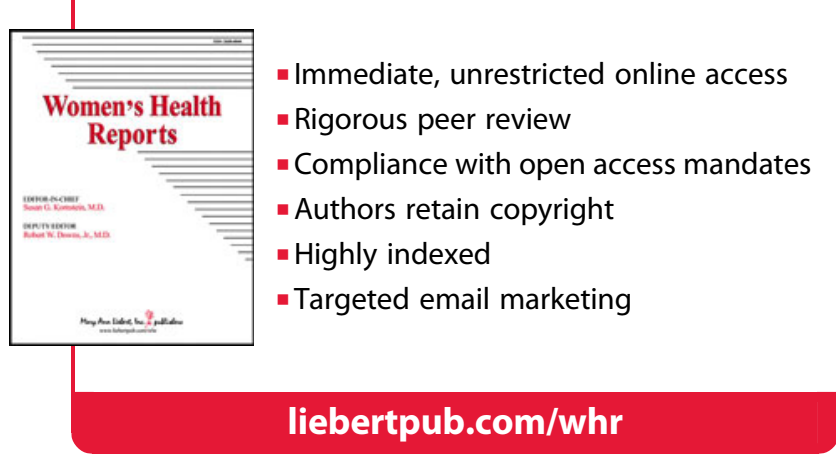

